\section{Assessment of responsiveness of the musculoskeletal component of SLE-DAS in an independent cohort}

We read with interest the report by Jesus et al on the Systemic Lupus Erythematosus Disease Activity Score (SLE-DAS). ${ }^{1}$ We agree that the systemic lupus erythematosus disease activity index 2000 (SLEDAI-2K) in its original form has limitations as a disease activity tool. Since each organ manifestation can only be graded as present or absent, it is unable to differentiate between mild, moderate or severe disease activity, nor partial response in any feature.

Our group is researching outcome measures in musculoskeletal SLE. We published a previous study of responsiveness of various musculoskeletal outcome measures in patients treated with glucocorticoids. ${ }^{2}$ As expected, the musculoskeletal component of the SLEDAI-2K was less responsive than the physician visual analog scale (physician VAS), musculoskeletal component of the British Isles Lupus Assessment Group (BILAG) index or ultrasound, all of which allow for various grades of severity.

The musculoskeletal component of the new SLE-DAS should, in theory, improve its responsiveness as it includes a term for swollen joint count as a continuous variable. We therefore calculated musculoskeletal component of new SLE-DAS tool in our previously published data set of 20 patients. These patients had objective synovitis, were treated with $120 \mathrm{mg}$ of intramuscular depomedrone and assessed at 0 and 4 weeks. We calculated the musculoskeletal component of the SLE-DAS using the terms:

\section{$3.132 x$ (Presence of Arthritis Yes/No) + \\ $0.454 x$ (swollen joint count in 28 joints)}

As in our publication, change was assessed using Wilcoxon signed-rank test. Effect size was calculated using standardised test statistic, $Z$, using the formula $r=Z / s q r t(n 1+n 2)$. Effect sizes were judged using Cohen's criteria as large $(\geq 0.5)$, medium $(\geq 0.3)$ or small $(\geq 0.1) .^{3}$

As expected, and like the other variables, the musculoskeletal SLE-DAS significantly reduced from median 13.4 (IQR 12.614.7) to median 6.2 (IQR $0.0-12.5, \mathrm{p}=0.001$ ). The effect size was large at -0.548 . As predicted by its developers, this was superior to the musculoskeletal component of SLEDAI-2K which had an effect size of -0.418 . However, the musculoskeletal SLEDAS was not as responsive as the musculoskeletal component of the BILAG index (-0.576), which could be graded as improving, or physician VAS $(-0.599)$, which was a continuous variable. However, the disadvantage of these two variables is that they are heavily dependent on the training and expertise of the assessors. Measures based on joint counts like SLE-DAS may show more robust inter-reader reliability.

There is another crucial feature of musculoskeletal lupus in which the BILAG index and physician VAS are advantageous: subclinical synovitis. We recently showed that in patients with SLE presenting with inflammatory joint pain, $38 \%$ had swollen joints. However, a further $27 \%$ had ultrasound-proven synovitis without a swollen joint. Ultrasound-only synovitis was clinically significant, being associated with worse serology, and patientreported and physician-reported outcomes. ${ }^{4}$ Since the musculoskeletal component of SLE-DAS is based entirely on swollen joint count, it would not be able to detect or monitor disease activity in this subgroup.

In summary, we validate the superior responsiveness of the musculoskeletal component of SLE-DAS compared with the SLEDAI-2K in an independent longitudinal study. However, our data suggest that a better outcome measure focusing on this specific organ system could be designed.

\section{Sabih-Ul Hassan $\odot,^{1,2}$ Khaled Mahmoud, ${ }^{1,2}$ Edward M Vital ${ }^{1,2}$}

${ }^{1}$ Department of Rheumatology, University of Leeds, Leeds Institute of Rheumatic and Musculoskeletal Medicine, Leeds, UK

${ }^{2}$ Leeds Biomedical Research Centre, Leeds, UK

Correspondence to Dr Edward M Vital,University of Leeds Leeds Institute of Rheumatic and Musculoskeletal Medicine, Leeds, Leeds, UK; e.m.j.vital@leeds.ac.uk

Contributors KM and EMV collected the data. SUH analysed the data and wrote the manuscript. All authors reviewed the manuscript and approved the final version.

Funding This study was funded by NIHR (grant no: CS-2013-13-032).

Competing interests None declared.

Patient consent for publication Not required.

Provenance and peer review Not commissioned; internally peer reviewed.

Data sharing statement Readers may contact the corresponding author to discuss access to the data.

(c) Author(s) (or their employer(s)) 2020. No commercial re-use. See rights and permissions. Published by BMJ.

\section{Check for updates}

To cite Hassan S-U, Mahmoud K, Vital EM. Ann Rheum Dis 2020;79:e51.

Received 20 March 2019

Accepted 24 March 2019

Published Online First 25 April 2019

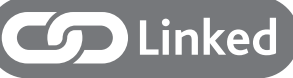

- http://dx.doi.org/10.1136/annrheumdis-2019-215430

Ann Rheum Dis 2020;79:e51. doi:10.1136/annrheumdis-2019-215411

\section{ORCID iD}

Sabih-Ul Hassan http://orcid.org/0000-0001-6635-8011

\section{REFERENCES}

1 Jesus D, Matos A, Henriques C, et al. Derivation and validation of the SLE disease activity score (SLE-DAS): a new SLE continuous measure with high sensitivity for changes in disease activity. Ann Rheum Dis 2019;78:365-71.

2 Mahmoud K, Zayat AS, Yusof Y, et al. Responsiveness of clinical and ultrasound outcome measures in musculoskeletal systemic lupus erythematosus. Rheumatology 2019;62.

3 Cohen J. Statistical power analysis for the behavioral sciences. Hillsdale, NJ: Erlbaum, 1988.

4 Zayat AS, Mahmoud K, Md Yusof MY, et al. Defining inflammatory musculoskeletal manifestations in SLE. Rheumatology. 2018;58:304-12.

5 Jesus D, Zen M, Doria A, et al. Response to: 'Assessment of responsiveness of the musculoskeletal component of SLE-DAS in an independent cohort', by Hassan, et al. Ann Rheum Dis 2020;79:e52. 